\title{
Computational Optimization for Structural Engineering Applications
}

\author{
Patrizia Trovalusci ${ }^{1} \cdot$ Giulio Maier $^{2}$ - Vincenzo Gattulli ${ }^{1}$ \\ Published online: 24 November 2020 \\ (c) Springer Science+Business Media, LLC, part of Springer Nature 2020
}

The present Special Issue (P. Trovalusci and G. Maier Editors; V. Gattulli co-Editor) collects selected and invited novel contributions that follow and extend some studies originally presented at the '9th International Conference on Computational Methods' (ICCM2018), held in Rome, Italy, in August 2018, with Professor Trovalusci as Chairman. The articles of the volume are devoted to recent advances in computational optimization for structural engineering applications focusing on theoretical, experimental, and practical aspects.

Computational optimization plays a key role in many fields of Structural Engineering, among these: structural identification of material parameters and damage detection; structural shape/topology optimization for design; optimization for collapse analysis aimed at risk mitigation and reliability of structures of cultural heritage. Focus is given on optimization problems that are nonlinear and non-convex and suffer from lack of uniqueness, the presence of global and local minima, and the strong dependency of the solution on the choice of the starting point in the minimization process. In many practical cases, moreover, the constraints and the derivatives cannot be known, and even the objective function may have unknown characteristics, and the constrained minimization problems are configured as black boxes. This scenario often requires the application of direct search methods of optimization and the call for the adoption of 'heuristic' or evolutionary optimization algorithms and learning techniques, that in many cases prove to be effective.

Each selected contribution of the Special Issue has undergone a deep review process, and only papers that have attracted positive recommendations, from at least two international referees, have been included. The papers provide engineering appli-

Patrizia Trovalusci

patrizia.trovalusci@uniroma1.it

Vincenzo Gattulli

vincenzo.gattulli@uniroma1.it

1 Department of Structural and Geotechnical Engineering, Sapienza University of Rome, Via Gramsci 53, 00197 Rome, Italy

2 Department of Structural Engineering, Politecnico di Milano, Piazza Leonardo da Vinci 32, 20133 Milan, Italy 
cations with special attention devoted to structural identification and diagnosis via reverse analysis, structural optimization methods for design, and mathematical programming for limit analysis of masonry structures. All articles are briefly introduced in the paragraphs below.

The paper by Amendola presents an optimized and convergent regularization procedure for the computation of the stress field exhibited by particle systems subjected to self-equilibrated short-range interactions. The analyzed systems of forces describe pair-interactions between lumped masses in 'atomistic' models for 2D bodies. Polyhedral stress functions are defined over arbitrary triangulations for 2D domains.

In the work by Bacigalupo et al., machine-learning techniques for the optimal design of acoustic metamaterials are presented. Novel functional applications of such research are expected in the optimal parametric design of smart tunable mechanical filters and directional waveguides. The present paper deals with the maximization of the amplitude of low-frequency band gaps, by proposing suitable numerical techniques to solve the associated optimization problems. Specifically, the feasibility and effectiveness of radial basis function networks and quasi-Monte Carlo methods for the interpolation of the objective functions of such optimization problems are discussed, and their numerical application to a specific acoustic metamaterial with tetrachiral microstructure is presented.

Bruggi and Taliercio present hierarchical infills for additive manufacturing via homogenization and optimization. The macroscopic properties of the hierarchical infill are computed through numerical homogenization once shape and the grading stages are given. A multi-material optimization problem is formulated to find the distribution of the prescribed discrete set of candidates that maximizes the structural stiffness of the object to be printed for a limited volume fraction. Numerical simulations, addressing the design of a self-supporting orthotropic rhombic infill and a stiff isotropic triangular one, are shown.

Catapano and Montemurro develop a multiscale two-level optimization strategy for composites where the optimization is exploited for finding the best strength of variable angle-tow composites subject to mechanical and manufacturing constraints. The strategy has a first step where the laminate strength is described through a laminatelevel failure criterion based on tensor invariants and on the first-order shear deformation theory; secondly the lay-up design phase makes use of quasi-trivial solutions and integrates a check on the first-ply failure in order to ensure the integrity of the whole laminate. The effectiveness of the strategy as well as of the proposed failure criterion is proven on some meaningful test cases.

Cocchetti and Rizzi investigate a static upper/lower thrust and kinematic work balance stationarity for least-thickness circular masonry arch optimization. This paper re-considers a recent analysis on the so-called "Couplet-Heyman problem" of least-thickness circular masonry arch structural form optimization and provides complementary and novel information and perspectives, specifically in terms of the optimization problem and its implications on the general understanding of the mechanics of masonry arches. A discontinuous deformation analysis (DDA) simulation tool and the operation of a new self-implemented complementarity problem/mathematical programming formulation, with a full matching of the achieved results, on all the arch characteristics in the critical condition of minimum thickness are adopted. 
In the work by Lofrano et al., a procedure for the optimal sensor placement in dynamic damage detection of beams is presented using a statistical approach. Attention is focused on the identification of the dynamic response of beam-like structures with uncertain damages. In particular, the non-localized damage is described using a Gaussian distributed random damage parameter. The procedure for selecting an optimal number of sensor placements has been presented based on the comparison between the probability of damage occurrence and the probability to detect damage, where the former can be evaluated from the known distribution of the random parameter, whereas the latter is evaluated exploiting the closed-form asymptotic solution provided by a perturbation approach. The presented case study shows the capability and reliability of the proposed procedure for detecting the minimum number of sensors such that the monitoring accuracy is not greater than a small control value.

Panettieri et al. present a work on multi-scale least-weight design of a wing-box through a global/local modeling approach. Such multi-scale optimization strategy is applied to realistic wing structure of a civil aircraft. Such procedure has two main characteristics. All design variables are taken into account, and a component-wise multi-scale modeling is proposed. Fully parametric and global and local finite element models are interfaced with an in-house genetic algorithm.

Pingaro et al. analyze a damage identification method for moderately thick cracked beams using an interdependent locking-free element. Static and dynamic problems of Timokshenko beams are presented where a cubic interpolation for the displacements and a quadratic one for the rotations is set. Mechanical behavior of the beam due to damaged zones and the identification of cracked zones in relation with the beam natural frequencies is presented.

In the work of Reggio et al., a stochastic multi-objective optimization procedure for exoskeleton structures is presented. The work is conceived as vibration control systems under seismic loading. The exoskeleton structure is assumed to be coupled to an existing primary inner structure for seismic retrofit. Two concurrent and competing objective functions are introduced, in order to take into account not only safety performance but also economic cost considerations. The resulting trade-off is solved searching the Pareto front by way of a controlled elitist genetic algorithm, derived from the non-dominated sorting genetic algorithm-II.

Tronci et al. present a self-automated operational modal analysis methodology to optimize modal parameter estimation. This work proves the necessity of abandoning identification approaches based on a single set of parameters for a long-term monitoring campaigns and to propose a semiautomated modal identification tool, where the user-defined parameters vary within an established range of values, that can be set independently of the user's expertise. Validation is proposed for modal analysis of a historic civil tower, and such results demonstrate the importance of multiple set of parameters when complex systems are considered.

Smarra et al. present a work on learning models for optimal control of seismicinduced vibrations in frame structures via the random forests method. Performance of neural networks, random forests and regression trees-based identification algorithms in producing reliable models exploiting historical data coming from a real structure are discussed. Peculiarities of each data-driven-based model emphasizing the strong potentialities of such approaches are highlighted, and it is shown in a simulative 
environment how, by slightly increasing the complexity of a model via random forests, we can reduce by half the active control effort with respect to the control computed exploiting regression trees-based models.

We hope these research issues will provide opportunities to identify and discuss future developments in the field of optimization in structural engineering applications. Our special thanks go to the Editor-in-Chief Franco Giannessi for inviting us to select and collect papers in a special issue of this journal and for assisting us in the editing work. Finally, we acknowledge also our colleague Nicholas Fantuzzi (University of Bologna (Italy)), who contributed to the volume with meticulous and careful review during its development.

Publisher's Note Springer Nature remains neutral with regard to jurisdictional claims in published maps and institutional affiliations. 